\title{
Direct Measurement of Osteolysis in Man
}

\author{
James W. Hansen, Gilbert S. Gordan, and Stanley G. Prussin \\ From the Department of Medicine, University of California, San Francisco 94122, \\ and the Department of Nuclear Engineering, University of California, \\ Berkeley, California 94720
}

A в S T R A C T Precise, direct measurement of bone calcium release $\left(v_{o_{-}}\right)$has been accomplished using a continuous tracer administration (CTA) technique. Dietary calcium $\left(96.97 \%{ }^{40} \mathrm{Ca}\right)$ is replaced by ${ }^{40} \mathrm{Ca}$ $\left(99.991 \%{ }^{40} \mathrm{Ca}\right)$ and blood levels of the naturally occuring isotope ${ }^{48} \mathrm{Ca}$ are monitored by neutron activation analysis as a function of time. ${ }^{48} \mathrm{Ca}$ abundance falls as this isotope is excreted and only partially replaced by release from bone. After a suitable period, an asymptotic abundance of ${ }^{48} \mathrm{Ca}$ in serum, $E$, is approached which is the fraction of the turnover rate of the rapidly exchangeable calcium pools coming from the skeleton $\left(E=v_{0} / v_{t}\right) . E$ is determined with a standard error of $2 \%$, providing a precise, sensitive index of $v_{0-} .13$ studies in three normal men and one postmenopausal woman receiving maintenance estrogen show large intersubject variations in parameters of calcium metabolism using both CTA and pulse tracer administration (PTA) plus balance techniques. Induced hypercalcemia results in a prolonged decrease in $v_{o-}$. Glucocorticoid therapy initially and consistently induces a marked hypercalciuria while effects on most other parameters of calcium kinetics are variable. In two men $E$ fell when testosterone was added to glucocorticoid treatment, consistent with the known antiosteolytic effect of androgens, despite the short period of study.

\section{INTRODUCTION}

Bone resorption (osteolysis) is of great importance in calcium and skeletal metabolism. Parathyroid hormone and calcitonin, respectively, increase and decrease osteolysis as their primary actions on bone to maintain blood calcium homeostasis; other hormones and

Dr. Hansen's present address is Reproduction Research Branch, National Institute of Child Health and Human Development, Bethesda, Md., 20014. (Please address reprint requests to Dr. Hansen.)

Received for publication 12 May 1972 and in revised form 6 September 1972. pharmacological agents (e.g. androgens, estrogens, and glucocorticoids) also affect bone breakdown.

Skeletal dynamics have been studied for many years using numerous techniques with variable degrees of success. In the investigation of skeletal kinetics many difficulties result from slow bone turnover (physiological destruction and replacement) and problems with representative measurements of skeletal composition in vivo. Techniques involving bone biopsy (1-3) have the disadvantages of representing only the portion of the skeleton sampled and of causing discomfort. Furthermore, translating static morphological observations $(2,3)$ into kinetic function may also lead to variation and error. Finally, quantitative in vivo roentgenography of the skeleton has not been sufficiently precise or reproducible to be useful in skeletal kinetic studies although recent advances have led to precise measurements at specific, local sites in the skeleton (4).

Since a large part of bone is mineral and more than $99 \%$ of total body calcium is in the skeleton, calcium metabolism is inseparably connected to skeletal dynamics. Consequently, studies of calcium in man have been useful in understanding and quantitating skeletal metabolism. Precise measurement of calcium in physiologic fluids and balance techniques (measuring net body retention) have been invaluable tools in studies of mineral metabolism. With the advent of radioactive and stable calcium tracers and precise detection techniques for them, kinetic studies of calcium metabolism have proved useful in understanding skeletal dynamics in vivo.

Most investigators of the kinetics of calcium metabolism have used a multicompartmental model similar to the widely accepted one shown in Fig. 1. Using the symbols of Aubert and Milhaud (5), $M_{1}$ and $M_{2}$ are, respectively, the masses of calcium in the rapidly exchangeable calcium pools where pool I $\left(M_{1}\right)$ includes serum calcium. For convenience, the rapidly exchangeable pools are referred to collectively as $\mathrm{Ca}_{e}$. Calcium flow is represented by the symbol $v: v_{I}$, rate 
of total calcium intake, $v_{a}$, net dietary calcium absorption rate, $v_{u}$, urinary calcium excretion rate, $v_{F}$, total fecal calcium excretion rate, $v_{f}$, net endogenous fecal excretion rate, $v_{12}$, exchange rate between pools $I$ and II, $v_{o+}$, accretion or rate of bone calcium deposition, $v_{0}$, osteolysis or rate of bone calcium resorption (including both morphologic bone breakdown and skeletal losses from mineral exchange), and $v_{t}$, rapidly exchangeable pool turnover, i.e.,

$$
v_{t}=v_{a}+v_{o_{-}}=v_{u}+v_{f}+v_{o+} .
$$

Calcium kinetics in this model are generally investigated by injecting a pulse of calcium tracer into the blood (pool I) and determining $M_{1}, M_{2}, v_{12}$, and $v_{t}$ from the disappearance of tracer from this pool as a function of time. By combining these parameters with data from a simultaneous balance study and tracer excretion measurements, the other parameters of calcium metabolism, $v_{0_{-}}, v_{o_{+}}$, and $v_{a}$, may be indirectly estimated.

In spite of a long history of attempts to study bone kinetics and the importance of osteolysis in health and disease, the very important parameter, $v_{0}$, has not been directly measured. Using pulse tracer administration (PTA) ${ }^{1}$ methods, $v_{o}$ can be estimated with a standard error of the order of $10 \%$ (a $95 \%$ confidence interval of $\pm 20 \%$ ). Because even small, persistent changes in $v_{0-}$ may result in skeletal disease, a more direct, precise determination of osteolysis in this model (Fig. 1) would seem desirable.

We have developed a calcium tracer technique to estimate bone calcium resorption, $v_{o_{-}}$, more directly and precisely than previously reported. This technique is a refinement of the continuous tracer administration (CTA) method used by Nordin, Smith, and Nisbet (6). The basic principle is to measure in $\mathrm{Ca}_{e}$ a stable, naturally occurring calcium tracer which is liberated from the skeleton by bone resorption after elimination (or marked reduction) of it in the diet. At equilibrium the fraction of rapidly exchangeable calcium of skeletal origin is therefore the fraction of the natural abundance of this tracer in $\mathrm{Ca}_{e}$, which is equivalent to $v_{0} / v_{t}$. Thus, a new parameter, $E=v_{o_{-}} / v_{t}$ or osteolysis normalized by turnover of $\mathrm{Ca}_{e}$, is defined and can be determined very precisely $\left(\sigma_{E}<2 \%\right)$ by direct measurement. In combination with other data, $E$ can then be used to calculate a relatively precise estimate of $v_{0}$.

To validate this CTA technique for measuring osteolysis, we carried out pilot studies in rats (7) and man (8) leading to the 13 studies in four adults re-

${ }^{1}$ Abbreviations used in this paper: AA, asymptotic abundance; CTA, chronic tracer administration; PTA, pulse tracer administration; TRP, tubular reabsorption of phosphate.

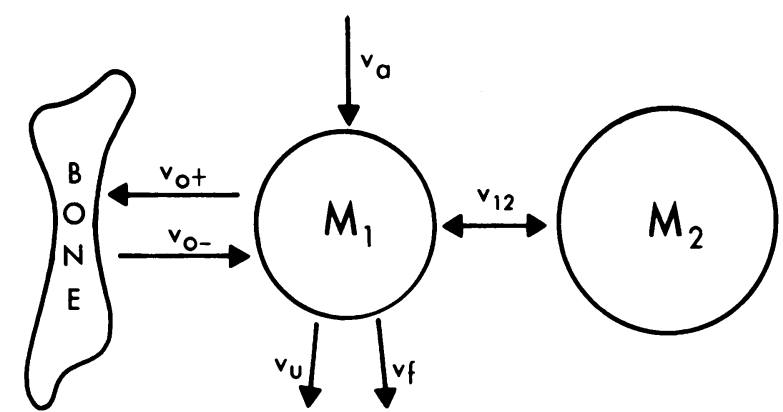

FIGURE 1 Model of in vivo calcium kinetics. Pools I and II represent rapidly exchangeable calcium, while "bone" represents the "stable" fraction of the skeleton. Pool size is represented by $M$ and calcium flow by $v$.

ported in this paper. In each case balance and PTA studies of calcium were also conducted. The validataion studies included normal adults in a basal state, in a hyperglucocorticoid state, and finally with appropriate androgen or estrogen added. One normal man was also studied after the stress of acute hypercalcemia.

\section{METHODS}

Theory. The model used is similar to that suggested by Harris and Heaney (9) (see Fig. 1) to describe in vivo calcium kinetics. By continuously marking dietary or skeletal calcium with a tracer, its abundance in the rapidly exchangeable pools $\left(\mathrm{Ca}_{e}\right)$ asymptotically approaches an abundance level at equilibrium $(E)$ directly proportional to the fraction of $v_{t}$ flowing from the labeled origin; this assumes that, during the experimental period, calcium metabolism is in a steady state and tracer abundance does not change significantly in either the diet or the "stable" skeleton. Since our goal is to measure bone calcium resorption as directly and precisely as possible, we used the naturally occurring ${ }^{48} \mathrm{Ca}$ as a uniformly distributed bone calcium tracer of known abundance and markedly reduced the level of this isotope in the diet. Serum ${ }^{48} \mathrm{Ca}$ abundance declined to an equilibrium level, $E$, as excreted tracer was only partially replaced with tracer from bone. Correction of $E$ for the small contribution of tracer from the diet results in $E_{c}$, which has the following relationship to $v_{o-}$ :

$$
v_{o_{-}} / v_{t}=E_{c} \text { or } v_{o_{-}}=E_{c} v_{t}
$$

where $E_{c}$ is expressed as the fraction of ${ }^{48} \mathrm{Ca}$ abundance in bone assumed in this work to be the same as the natural abundance of ${ }^{48} \mathrm{Ca}$. (10) (see Appendix A for the precise derivations of Eq. 2.)

In studies of calcium kinetics, the steady-state assumption is generally accepted under controlled conditions of constant intake. The new assumption necessary in the present work is that tracer abundance in the skeleton does not change significantly. Although calcium with reduced tracer content is deposited in bone during the experimental period, skeletal ${ }^{48} \mathrm{Ca}$ abundance is altered less than $0.025 \%$ per day in our studies. Furthermore, in view of evidence for random bone calcium resorption via osteocytic osteolysis (11) and the improbability that exchange processes and osteoclastic osteolysis occur specifically at sites of label concentration or deficiency, it is likely that tracer resorption would approximate that from a homogeneous pool in spite of gross morphological inhomogeneity (12). This concept of random resorption of label from bone is also supported by the observation that

Direct Measurement of Osteolysis in Man 


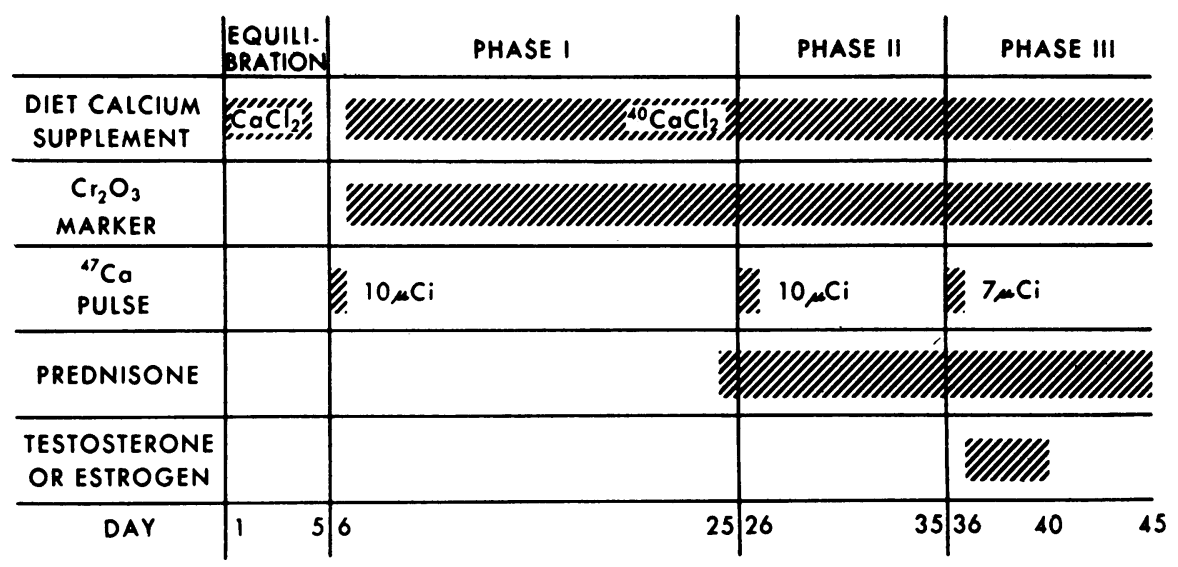

FIGURE 2 General protocol of clinical studies outlining differences between phases. Except where mass number is indicated $\left({ }^{40} \mathrm{Ca} ;{ }^{47} \mathrm{Ca}\right)$, the symbol $\mathrm{Ca}$ represents calcium of natural isotopic composition.

in rats labeled with radioactive calcium and maintained on a near zero calcium intake, the sp act of bone and serum calcium are the same after equilibration for a few weeks (13).

Clinical material. Three white males, ages 26 (P. H.), 29 (S. P.), and 36 (B. D.), and one 49-yr-old surgically menopausal Negro female (E. W.) maintained on estrogen $(2.5 \mathrm{mg}$ Premarin per day) were studied. No other drugs or medications were being taken by any of the subjects. By history and physical examination all are normal adults. P. H. is of medium build with athletic features; S. P. is small in stature with fine features, and B. D. is rather stocky. The history of these subjects indicated variable daily calcium intake, ranging from 600 to $800 \mathrm{mg}$ daily for S. P., B. D. and E. W. and 800-1,000 $\mathrm{mg} /$ day for P. H. Base-line laboratory tests, including serum calcium, phosphorus, alkaline phosphatase, protein electrophoresis, urea nitrogen, creatinine, carotene, cholesterol, glucose, sodium, potassium, chloride, and total carbon dioxide content, were within normal limits for all subjects. $12 \mathrm{~h}$, overnight urine excretion revealed normal calcium (see footnote 2) and creatinine content, as well as normal calculated values for tubular reabsorption of phosphate (TRP) and creatinine clearance. Complete blood counts and urinalyses were also normal. Fusion of epiphyses and absence of skeletal disease were confirmed by roentgenography. On X-ray examination the bones of the black female subject appeared quite dense.

Clinical studies. Each subject was given a fixed, low calcium ( $<66 \mathrm{mg} /$ day) diet supplemented to approximately their own normal calcium and phosphorus intake with calcium chloride and sodium hydrogen phosphate solutions divided with meals. A maintenance multivitamin tablet was given daily, and methylcellulose was used to increase bulk in the low residue diet. The subjects maintained their normal daily activity and meal schedules. The metabolic kitchen of the General Clinical Research Ward, Moffitt Hospital, University of California, San Francisco, provided the carefully prepared and weighed food which was completely consumed.

$312 \mathrm{~h}$ overnight urinary calcium excretion in these subjects before kinetic studies were started was between $50-150 \mathrm{mg}$. In view of only minimal effects of diurnal variation on urinary calcium in 12-h collections (see Appendix D), these excretion rates are well within the normal range of excretion rates found by Neer, Berman, Fisher, and Rosenberg (24), 38-510 $\mathrm{mg} /$ day.
Chromium sesquioxide, $500 \pm 5 \mathrm{mg}$, was given three times a day with meals as a continuous fecal marker $(8,14)$ during each phase of the study.

In three of the four subjects studied the following protocol was used (see Fig. 2). After a 5 day equilibration period on the above diet, phase I commenced by intravenous injection of $10 \mu \mathrm{Ci}$ of ${ }^{47} \mathrm{Ca}$ in weighed solution and by changing the $\mathrm{CaCl}_{2}$ supplement solution to a solution of ${ }^{40} \mathrm{CaCl}_{2}$ prepared from enriched ${ }^{40} \mathrm{CaCO}_{3}\left(99.991 \%{ }^{40} \mathrm{Ca}\right.$ abundance) by dissolving in $\mathrm{HCl}$ and adjusting to $\mathrm{pH}$ of 5 with $\mathrm{NaOH}$. This phase lasted 20 days and gave base-line steady-state kinetic data. Prednisone, $22.5 \mathrm{mg} /$ day, was given during phases II and III. Calcium kinetics were studied for 10 days during phase II starting with the injection of $10 \mu \mathrm{Ci}^{47} \mathrm{Ca} 1$ day after beginning the prednisone. Phase III commenced with injection of $7 \mu \mathrm{Ci}$ ${ }^{47} \mathrm{Ca}$ and lasted for 10 days. During the first 5 days of this phase, the men received $100 \mathrm{mg}$ testosterone propionate in oil intramuscularly each day and the female increased her maintenance Premarin dose to $10 \mathrm{mg}$ each day.

In the fourth subject, B. D., a study of the effects of acute hypercalcemia was conducted between phases I and II. Phase I $a$ commenced with a $2.0 \mathrm{~g}, 10 \mathrm{~h}$ intravenous infusion of enriched ${ }^{40} \mathrm{CaCl}_{2}$. Serum calcium reached $14.9 \mathrm{mg} / 100 \mathrm{ml}$ at the end of the infusion and rapidly fell to $11.3 \mathrm{mg} / 100 \mathrm{ml}$ where it remained for 2 days. By this time, cumulative urine output in excess of previous levels totalled $2 \mathrm{~g}$ and serum calcium levels became normal. 2 days after beginning the infusion, $7 \mu \mathrm{Ci}{ }^{47} \mathrm{Ca}$ was injected and the effects of parenteral calcium loading on calcium kinetics were studied over a 15 day period.

Three types of studies were carried out simultaneously during each phase. Measurement of intake and collection of urine and feces with the use of a continuous fecal marker allowed classical balance calculations. PTA of ${ }^{47} \mathrm{Ca}$ enabled classical kinetic studies (15). Finally, CTA studies were possible, using the ${ }^{48} \mathrm{Ca}$ tracer with the results being compared to balance and PTA studies to validate this technique.

Laboratory procedures. Fecal specimens were homogenized with $10 \%$ concentrated $\mathrm{HNO}_{3}$ and a sample digested in Kjeldahl flasks with $\mathrm{HNO}_{3}$ and then $\mathrm{HClO}_{4}$ to determine total fecal $\mathrm{Ca}$ and $\mathrm{Cr}$ content. The acid supernatant of the fecal homogenate was treated the same as urine and serum for ${ }^{47} \mathrm{Ca}$ sp act and ${ }^{48} \mathrm{Ca}$ abundance determinations. Acidified urine was filtered through ashless paper and a sample taken for total calcium determination; the balance was processed 
the same as serum for calcium tracer measurements. Calcium was precipitated from serum, urine, and the acid supernatant of fecal homogenates by the addition of saturated $\left(\mathrm{NH}_{4}\right)_{2} \mathrm{C}_{2} \mathrm{O}_{4}$ solution. The rinsed $\mathrm{CaC}_{2} \mathrm{O}_{4}$ precipitate was redissolved in concentrated $\mathrm{HNO}_{3}$ and samples taken for tracers, ${ }^{47} \mathrm{Ca}$ and ${ }^{48} \mathrm{Ca}$. On representative days, food portions identical with those consumed were homogenized and samples digested in $\mathrm{HNO}_{3}$ and $\mathrm{HClO}_{4}$ for dietary calcium determination. Calcium concentrations of solutions were measured, using a modification of the emission spectrophotometric technique of Loken, Teal, and Eisenberg (16) with a SD of $0.8 \% .{ }^{47} \mathrm{Ca}$ activity was determined to within $2 \%$ (SD) using a NaI (Tl) scintillation counting system with an absolute efficiency of $18 \%$ and background of $27.5 \mathrm{cpm}$ utilizing the energy range $0.70-1.44 \mathrm{MeV} .{ }^{48} \mathrm{Ca}$ abundance was determined by neutron activation analysis with a SD ranging from $2 \%$ to $4 \%$ (17). Chromium levels were also determined by flame emission spectrophotometry (8).

Calculations (8). For PTA studies the first 10 days of data were fitted with the equation

$$
X=C_{1} e^{-\lambda_{1} t}+C_{2} e^{-\lambda_{2} t},
$$

and used to calculate the parameters of calcium metabolism (18). $X$ is the measured tracer sp act or abundance in pool I as a function of time. For comparison, calculations were also made by fitting with a three component equation:

$$
X=C_{1} e^{-\lambda_{1} t}+C_{2} e^{-\lambda_{2} t}+C_{3} e^{-\lambda_{3} t}
$$

Because of the uncertainties in interpretation of the third component, (see Discussion section) the flow parameters of calcium kinetics $(v)$ were calculated using only Eq. 3 and not Eq. 4.

Using the model in Fig. 1, a two component exponential equation with an additional constant term was used to fit CTA data:

$$
X=D_{1} e^{-\lambda_{1} t}+D_{2} e^{-\lambda_{2} t}+E,
$$

where $\lambda_{1}$ and $\lambda_{2}$ are the same as those in PTA studies. Because of scatter in the data, large errors in $D_{1}, D_{2}, \lambda_{1}, \lambda_{2}$, and $\mathrm{E}$ result when all are estimated from the fit.

Therefore, by using $\lambda_{1}$ and $\lambda_{2}$ from PTA data analysis, a more precise value for $\mathrm{E}$ can be obtained. However, if "equilibrium" is reached within the desired precision, then the first two terms of Eq. 5 become insignificant and $X$ approaches $E$ as a nearly model-independent estimate of this parameter of calcium kinetics. In using Eq. 5, dietary contributions of tracer to $X$ were corrected for as described in Appendix A. Thus $E_{c}$ is obtained for use in Eq. 2.

Computations were made on an IBM 360/50 computer, using variance weighted least-squares procedures to fit the data to the equations and obtain error estimates of the parameters. Standard statistical formula were used to quantify the precision of balance data. Errors $\left(\sigma_{P}\right)$ of the derived parameters of physiological interest $\left(P=v, M, E\right.$, or $\left.E_{c}\right)$ were estimated from errors $\left(\sigma_{p}\right)$ in the component parameters ( $p=C, \lambda$, or other component measurements) according to the following formulation:

$$
\sigma_{P^{2}}=\sum_{i=1}^{n}\left[P-f\left(p_{1}, p_{2}, \cdots p_{i}+\sigma_{p_{i}}, \cdots p_{n}\right)\right]^{2}
$$

where $P=f\left(p_{1}, p_{2}, \cdots p_{n}\right)$ (see footnote 3 ). Precision in this

${ }^{3} \mathrm{Eq} .6$ gives $\sigma_{P^{2}}$ values which agree within $1 \%$ of those obtained using the following expression:

$$
\sigma P^{2}=\sum_{i=1}^{n}(\partial P / \partial P)^{2} \sigma_{p_{i}}{ }^{2} .
$$

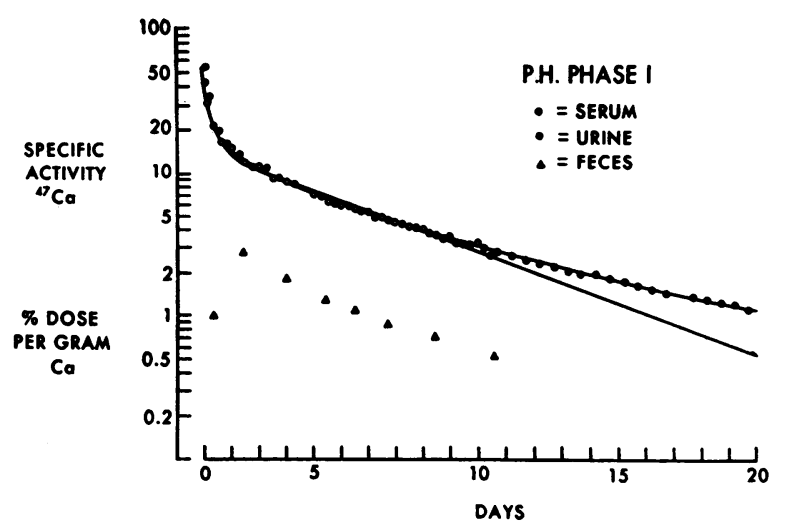

Figure 3 Representative data from PTA studies in P. H. Errors are included within the sizes of the points.

paper is expressed as $\pm 1 \mathrm{SD}$ of the mean for the derived parameters for each subject and for those directly measured parameters of physiologic interest, e.g. $v_{u}, v_{F}, v_{I}$, etc. Errors in the raw data, e.g. calcium concentration, ${ }^{47} \mathrm{Ca}$ sp act, ${ }^{48} \mathrm{Ca}$ abundance, etc., are \pm 1 estimated SD of the determination.

\section{RESULTS}

Figs. 3 and 4 show representative data for PTA and CTA studies, respectively, from 20 days of base-line study during phase $\mathrm{I}$ in $\mathrm{P}$. $\mathrm{H}$. Although the plotted \pm 1 $\sigma$ errors appear smaller for the ${ }^{47} \mathrm{Ca}$ determinations in PTA studies than they do for the ${ }^{48} \mathrm{Ca}$ measurements in CTA studies, they are in fact relatively similar, i.e., $3 \%$ and $4 \%$, respectively. The apparent discrepancy is due to the difference in the magnitude of change in the measured variable and the fact that ${ }^{47} \mathrm{Ca}$ data are plotted similogarithmically while natural ${ }^{48} \mathrm{Ca}$ abundance data are plotted linearly. The best weightedleast-squares fits using two and three component exponential Eqs. 3 and 4, are drawn through the PTA data. The three-component equation results in the

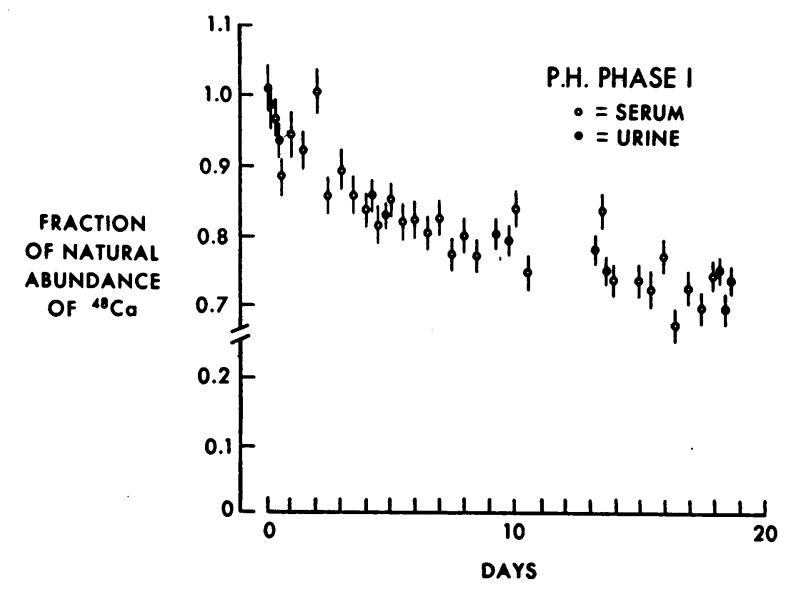

FIliure 4 Representative data from CTA studies in P. H. The vertical bar represents a range of mean $\pm 1 \sigma$.

Direct Measurement of Osteolysis in Man 
TABLE I

Parameters of Calcium Kinetics Using a Two-Compartment

\begin{tabular}{|c|c|c|c|c|c|c|c|c|c|c|c|c|}
\hline Subject & Age & Sex & $\mathbf{H t}$ & Wt & Phase & $M_{1}$ & $M_{2}$ & $M_{1}+M_{2}$ & $v_{12}$ & $v_{t}$ & $v_{u}$ & vs \\
\hline \multirow[t]{4}{*}{ B. D. } & 36 & $\mathbf{M}$ & 1.71 & 75 & I & $2.19 \pm 0.09$ & $2.91 \pm 0.12$ & $5.10 \pm 0.14$ & $4.31 \pm 0.53$ & $0.729 \pm 0.027$ & $0.436 \pm 0.011$ & $0.106 \pm 0.003$ \\
\hline & & & & & I $a$ & $2.52 \pm 0.14$ & $3.04 \pm 0.20$ & $5.57 \pm 0.22$ & $3.12 \pm 0.52$ & $0.665 \pm 0.043$ & $0.355 \pm 0.022$ & $0.102 \pm 0.005$ \\
\hline & & & & & II & $2.88 \pm 0.20$ & $2.35 \pm 0.29$ & $5.23 \pm 0.36$ & $1.94 \pm 0.54$ & $0.804 \pm 0.064$ & $0.606 \pm 0.019$ & $0.086 \pm 0.007$ \\
\hline & & & & & III & $2.24 \pm 0.18$ & $3.07 \pm 0.34$ & $5.31 \pm 0.39$ & $2.99 \pm 0.76$ & $0.785 \pm 0.084$ & $0.557 \pm 0.045$ & $0.072 \pm 0.006$ \\
\hline \multirow[t]{3}{*}{ S. P. } & 29 & $\mathbf{M}$ & 1.71 & 70 & I & $1.90 \pm 0.10$ & $2.83 \pm 0.15$ & $4.73 \pm 0.17$ & $3.52 \pm 0.51$ & $0.835 \pm 0.036$ & $0.493 \pm 0.010$ & $0.071 \pm 0.003$ \\
\hline & & & & & II & $2.10 \pm 0.09$ & $2.69 \pm 0.14$ & $4.79 \pm 0.16$ & $3.35 \pm 0.45$ & $0.980 \pm 0.036$ & $0.733 \pm 0.038$ & $0.052 \pm 0.002$ \\
\hline & & & & & III & $2.32 \pm 0.08$ & $2.88 \pm 0.16$ & $5.20 \pm 0.19$ & $2.36 \pm 0.26$ & $1.043 \pm 0.044$ & $0.776 \pm 0.021$ & $0.045 \pm 0.002$ \\
\hline \multirow[t]{3}{*}{ P. H. } & 26 & $\mathbf{M}$ & 1.82 & 72 & I & $2.18 \pm 0.09$ & $3.41 \pm 0.16$ & $5.59 \pm 0.19$ & $3.68 \pm 0.43$ & $1.021 \pm 0.040$ & $0.328 \pm 0.011$ & $0.122 \pm 0.004$ \\
\hline & & & & & II & $2.33 \pm 0.09$ & $2.93 \pm 0.16$ & $5.26 \pm 0.19$ & $3.08 \pm 0.38$ & $1.067 \pm 0.045$ & $0.405 \pm 0.017$ & $0.103 \pm 0.004$ \\
\hline & & & & & III & $2.23 \pm 0.12$ & $3.32 \pm 0.22$ & $5.55 \pm 0.26$ & $3.10 \pm 0.47$ & $1.071 \pm 0.059$ & $0.411 \pm 0.024$ & $0.102 \pm 0.005$ \\
\hline \multirow[t]{3}{*}{ E. W. } & 49 & F & 1.61 & 70 & I & $1.79 \pm 0.06$ & $1.83 \pm 0.09$ & $3.62 \pm 0.10$ & $2.07 \pm 0.27$ & $0.445 \pm 0.019$ & $0.170 \pm 0.007$ & $0.066 \pm 0.002$ \\
\hline & & & & & II & $1.65 \pm 0.08$ & $1.89 \pm 0.08$ & $3.54 \pm 0.09$ & $4.10 \pm 0.66$ & $0.506 \pm 0.018$ & $0.223 \pm 0.010$ & $0.049 \pm 0.001$ \\
\hline & & & & & III & $1.52 \pm 0.07$ & $2.08 \pm 0.10$ & $3.60 \pm 0.11$ & $2.89 \pm 0.44$ & $0.491 \pm 0.022$ & $0.225 \pm 0.010$ & $0.051 \pm 0.002$ \\
\hline
\end{tabular}

Phase: I, base line; I $a$, after Ca infusion; II, during prednisone; III, during prednisone + androgen or estrogen.

$E_{c}, E$ corrected for diet ${ }^{4} \mathrm{Ca}$. Units of $: M$, grams; $v$, grams/day; $k$, day ${ }^{-1} ; E_{\varepsilon}$, (none). Ht, meters: wt, kilograms.

E. W. maintained on $2.5 \mathrm{mg}$ Premarin in all phases. Errors given as $\pm 1 \mathrm{SE}$.

* $E_{0}$ was calculated primarily from CTA data and equation (5) resulting in the consistently smaller error in $v_{v_{-}}=E_{c} v_{t}$ as compared to $v_{v_{-}}$determined from PTA and balance data.

best fit throughout the 20 day phase. However, the two component equation fit to the first 10 days of data represents this period fairly well.

The sensitivity and precision of the ${ }^{47} \mathrm{Ca} \mathrm{sp}$ act determinations are demonstrated by the first 10 days of data for E. W. shown in Fig. 5 where the $1 \sigma$ errors of the determinations are included in the sizes of the points. Daily fluctuations in ${ }^{47} \mathrm{Ca}$ sp act can be seen, being higher than expected at the beginning of the day after a night of fasting than at the end of the previous day and then declining throughout the day as the ${ }^{47} \mathrm{Ca}$ is diluted by dietary calcium in addition to normal biological excretion. This daily stepwise fluctuation is seen because of a slight lag in equilibration within $\mathrm{Ca}$.

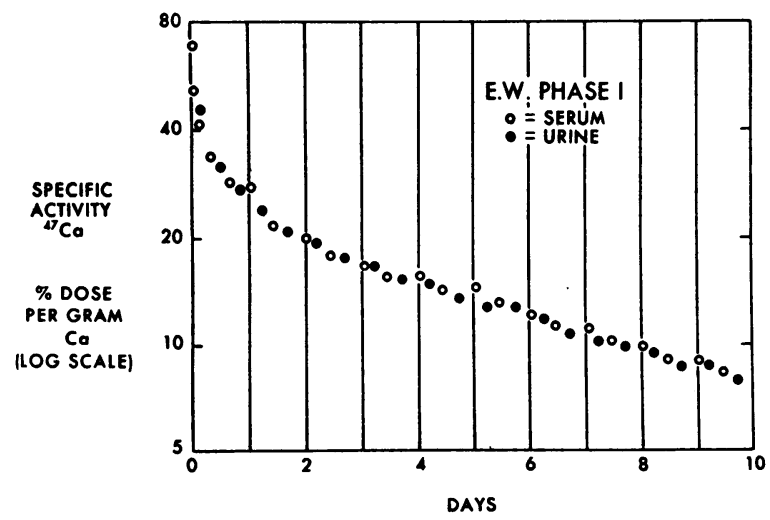

Figure 5 PTA data from serum and urine samples of $E$. W. Note that sensitivity and precision of the data are demonstrated by the expected diurnal discontinuities in the curve.
Table I summarizes the parameters of calcium kinetics of physiological interest obtained in these studies and compares $v_{0}$ calculated with and without utilizing CTA data. Phase I values are compared with normal values from other studies in Table II. The effects of glucocorticoids on calcium metabolism are compared in Table III with effects observed by others. Table IV shows various values obtained for decay constants $\left(\lambda_{i}\right)$ in PTA studies of calcium kinetics depending on the model used to define the rapidly exchangeable calcium pools, $\mathrm{Ca}_{e}$. The advantages of using CTA data to calculate $v_{0}$ are demonstrated in Table $\mathrm{V}$ which compares errors in the various calcium parameters calculated using different combinations of CTA, PTA, and balance data.

\section{DISCUSSION}

Theoretical considerations. Precise, direct determination of $E_{c}$, the fraction of rapidly miscible calcium pool turnover rate due to osteolysis, is the unique feature of this study. It can be precisely determined using CTA data in conjunction with parameters obtained from simultaneous PTA studies.

The validity of using $E_{c}$ to calculate $v_{0}$ is supported by the observation that $v_{0}$ values calculated from either PTA or CTA data do not differ significantly. This consistency is maintained regardless of perturbations-induced in each phase. In addition to the direct nature of the measurement of $v_{0}$ - afforded by using $E_{c}$, another distinct advantage of the CTA method is the increased precision in $v_{0}$. The PTA and balance data combine to give values of $v_{0}$ with $\mathrm{SE}$ of $6.5-14 \%$ 


\begin{tabular}{|c|c|c|c|c|c|c|c|c|}
\hline$v_{0+}$ & $v_{0_{-}}=E_{c} v_{t} *$ & $v_{v_{-}}=v_{o_{+}}-v_{\Delta}$ & $v_{a}=\left(1-E_{c}\right) v_{t}$ & $v_{a}=v_{t}-v_{o_{+}}+v_{\Delta}$ & $v_{F}=\frac{\mathrm{Ca} P}{\Delta t}$ & $v_{\Delta}=v_{1}-v_{F}-v_{u}$ & $k_{o_{-}}=\frac{v_{o_{-}}}{M_{1}+M_{2}}$ & $E_{c} *$ \\
\hline $0.186 \pm 0.029$ & $0.463 \pm 0.018$ & $0.417 \pm 0.028$ & $0.266 \pm 0.011$ & $0.312 \pm 0.008$ & $0.452 \pm 0.007$ & $-0.231 \pm 0.013$ & $0.0908 \pm 0.0043$ & $0.635 \pm 0.008$ \\
\hline $0.207 \pm 0.048$ & $0.368 \pm 0.025$ & $0.426 \pm 0.052$ & $0.298 \pm 0.021$ & $0.239 \pm 0.029$ & $0.520 \pm 0.029$ & $-0.219 \pm 0.036$ & $0.0661 \pm 0.0053$ & $0.553 \pm 0.012$ \\
\hline $0.112 \pm 0.067$ & $0.463 \pm 0.038$ & $0.514 \pm 0.065$ & $0.341 \pm 0.027$ & $0.290 \pm 0.014$ & $0.453 \pm 0.012$ & $-0.402 \pm 0.023$ & $0.0885 \pm 0.0094$ & $0.576 \pm 0.005$ \\
\hline $0.156 \pm 0.096$ & $0.465 \pm 0.051$ & $0.499 \pm 0.085$ & $0.320 \pm 0.034$ & $0.286 \pm 0.012$ & $0.443 \pm 0.010$ & $-0.342 \pm 0.046$ & $0.0876 \pm 0.0116$ & $0.592 \pm 0.007$ \\
\hline $0.271 \pm 0.028$ & $0.535 \pm 0.024$ & $0.532 \pm 0.047$ & $0.301 \pm 0.015$ & $0.303 \pm 0.030$ & $0.425 \pm 0.029$ & $-0.261 \pm 0.031$ & $0.1131 \pm 0.0065$ & $0.640 \pm 0.008$ \\
\hline $0.195 \pm 0.052$ & $0.592 \pm 0.023$ & $0.660 \pm 0.040$ & $0.388 \pm 0.016$ & $0.320 \pm 0.016$ & $0.389 \pm 0.016$ & $-0.465 \pm 0.041$ & $0.1236 \pm 0.0064$ & $0.604 \pm 0.008$ \\
\hline $0.223 \pm 0.049$ & $0.606 \pm 0.026$ & $0.678 \pm 0.045$ & $0.437 \pm 0.019$ & $0.365 \pm 0.008$ & $0.337 \pm 0.008$ & $-0.455 \pm 0.022$ & $0.1165 \pm 0.0066$ & $0.581 \pm 0.004$ \\
\hline $0.571 \pm 0.042$ & $0.727 \pm 0.030$ & $0.662 \pm 0.043$ & $0.294 \pm 0.015$ & $0.359 \pm 0.017$ & $0.625 \pm 0.016$ & $-0.090 \pm 0.019$ & $0.1301 \pm 0.0070$ & $0.712 \pm 0.009$ \\
\hline $0.560 \pm 0.048$ & $0.708 \pm 0.032$ & $0.663 \pm 0.053$ & $0.359 \pm 0.019$ & $0.404 \pm 0.029$ & $0.566 \pm 0.029$ & $-0.103 \pm 0.033$ & $0.1346 \pm 0.0078$ & $0.664 \pm 0.011$ \\
\hline $0.558 \pm 0.064$ & $0.645 \pm 0.037$ & $0.641 \pm 0.073$ & $0.426 \pm 0.026$ & $0.430 \pm 0.043$ & $0.543 \pm 0.043$ & $-0.084 \pm 0.049$ & $0.1162 \pm 0.0087$ & $0.602 \pm 0.010$ \\
\hline $0.209 \pm 0.020$ & $0.215 \pm 0.010$ & $0.135 \pm 0.021$ & $0.230 \pm 0.011$ & $0.310 \pm 0.010$ & $0.413 \pm 0.009$ & $+0.075 \pm 0.012$ & $0.0594 \pm 0.0032$ & $0.484 \pm 0.011$ \\
\hline $0.234 \pm 0.021$ & $0.221 \pm 0.009$ & $0.203 \pm 0.029$ & $0.285 \pm 0.011$ & $0.303 \pm 0.022$ & $0.403 \pm 0.022$ & $+0.031 \pm 0.025$ & $0.0624 \pm 0.0029$ & $0.437 \pm 0.007$ \\
\hline $0.215 \pm 0.024$ & $0.219 \pm 0.010$ & $0.207 \pm 0.028$ & $0.272 \pm 0.012$ & $0.284 \pm 0.018$ & $0.423 \pm 0.018$ & $+0.008 \pm 0.020$ & $0.0608 \pm 0.0035$ & $0.447 \pm 0.006$ \\
\hline
\end{tabular}

for each subject. This error in $v_{\sigma_{-}}$is reduced to 3.5$4.5 \%$ by utilizing results of CTA studies. Even then, the largest portion of this error is from errors in $v_{t}$ and not from those in $E_{c}$.

$E_{c}$ may be viewed as the rate of osteolysis normalized by turnover rate of $\mathrm{Ca}_{e}$, i.e. $E_{c}=v_{o-} / v_{t}$. The validity of $E_{c}$ as a normalized measure of osteolysis is borne out by agreement within error with $v_{o} / v_{t}$ calculated from the results of PTA and balance data. Such normalization often increases the sensitivity of a measurement as long as the normalizing factor does not also change in direct proportion to the measurement as a result of the perturbation being studied. For example, $E_{c}$ changed significantly between phases I and II in B. D., P. H., and E. W. while $v_{o}$ did not appear to be altered. The sensitivity of $E_{c}$ in the present studies is also enhanced by the small error of the determination in contrast to the larger error in $v_{0-} / v_{t}$ calculated from PTA and balance data.
Other possible normalizing factors could be body weight, body surface area, size of $\mathrm{Ca}_{e}\left(M_{1}+M_{2}\right)$, etc. With the similarity in weight of these individuals in the present study there was little advantage to normalizing with any of these factors. Furthermore, use of these factors with their imprecision would also tend to increase the error in the normalized parameter and decrease its sensitivity.

Classical kinetic studies using PTA have been conducted, using a number of different models for the rapidly miscible compartments, $\mathrm{Ca}_{e}$ (see Table IV). In early studies (19-21) $\mathrm{Ca}_{e}$ was of ten simplified to a single compartment, but it became apparent that at least two compartments were required to fit the first 10 days of data and compensate for excessive excretion of tracer during the intitial mixing phases (14). Three compartments have also been used $(20,22,23)$ (including an additional very rapidly turning-over pool) which does not significantly affect the parameters of

TABLE II

Comparison of Calcium Kinetic Data

\begin{tabular}{cccccccc}
\hline & & & & \multicolumn{4}{c}{ Present study } \\
\cline { 5 - 8 } Parameter & $\begin{array}{c}\text { Wajchenberg et al. (23) } \\
\text { Range, } n=4\end{array}$ & $\begin{array}{c}\text { Neer et al. (24) } \\
\mathrm{m} \pm \sigma, n=10\end{array}$ & B. D. & S. P. & P. H. & E. W. \\
\hline$v_{a}$ & $0.35-$ & 0.82 & $0.411 \pm 0.155$ & 0.266 & 0.301 & 0.294 & 0.230 \\
$v_{f}$ & $0.14-$ & 0.44 & $0.167 \pm 0.032$ & 0.106 & 0.071 & 0.122 & 0.066 \\
$v_{u}$ & $0.05-$ & 0.38 & $0.274 \pm 0.118$ & 0.436 & 0.493 & 0.328 & 0.170 \\
$v_{o+}$ & $0.38-$ & 1.22 & $0.534 \pm 0.270$ & 0.186 & 0.271 & 0.571 & 0.209 \\
$v_{o-}$ & $0.25-$ & 1.14 & $0.568 \pm 0.253$ & 0.463 & 0.535 & 0.727 & 0.215 \\
\hline
\end{tabular}

$v_{i}$ in units of $\mathrm{g} /$ day. 
TABLE III

Glucocorticoid Effects on Calcium

\begin{tabular}{ccccccc}
\hline Parameter & Eisenberg (29) & Wajchenberg et al. (23) & B. D. & S. P. & P. H. & E. W. \\
\hline$v_{u}$ & + & + & + & + & + & + \\
$v_{f}$ & & $-(\alpha)$ & - & - & - & - \\
$v_{a}$ & & 0 & + & + & + & + \\
$M_{1}+M_{2}$ & & 0 & $(+)$ & + & 0 & + \\
$v_{t}$ & + & + & 0 & 0 & 0 & 0 \\
$v_{o+}$ & + & - & 0 & + & 0 & 0 \\
$v_{\theta_{-}}$ & & - & - & - & 0 & 0 \\
$v_{\Delta}$ & & & - & - & - & - \\
$E_{c}$ & & & & & &
\end{tabular}

$P<0.05$ in present studies and those of Wajchenberg et al.

( ),$P>0.05$ but in direction of sign; $\alpha, v_{a} /$ dietary calcium intake; + , increased; - , decreased;

0 , no significant change.

TABLE IV

Comparison of Decay Constants, $\lambda_{i}$, Used in Various Models of $\mathrm{Ca}_{\mathrm{e}}$

\begin{tabular}{lccccc}
\hline \multicolumn{1}{c}{ Decay constant } & $\lambda_{0}$ & $\lambda_{1}$ & $\lambda_{2}$ & $\lambda_{3}$ & $\lambda_{4}$ \\
\hline Heaney et al. (19) & & & $\sim 0.10$ & & \\
Hansen (8) & & $\sim 3.5$ & $\sim 0.15$ & & \\
Wajchenberg et al. (23) & $\sim 60$ & $\sim 4$ & $\sim 0.2$ & & \\
Neer et al. (24) & 58 & 3.8 & 0.35 & 0.068 & \\
Harrison et al. (25) & & & 0.35 & 0.061 & 0.0016 \\
\hline
\end{tabular}

$\lambda_{i}$ in units of $(\text { day })^{-1}$. For details, see text; references cite sources of data for calculation of indicated decay constants.

bone calcium or balance kinetics derived from the model. However, Neer, Berman, Fisher, and Rosenberg (24) used PTA data for 20 days and included a slower fourth compartment in their analyses, as also considered by others $(5,20)$. In studies designed to look specifically

TABLE V

Percent Standard Errors Calculated from Data in Present Study*

\begin{tabular}{cccc}
\hline Parameters & $\begin{array}{c}\text { Balance data } \\
\text { +PTA data }\end{array}$ & $\begin{array}{c}\text { CTA data + PTA data } \\
\text { +Minimal balance data }\end{array}$ & $\begin{array}{c}\text { Balance data } \\
\text { +CTA data }\end{array}$ \\
\hline & $\%$ & $\%$ & $\%$ \\
$v_{\iota}$ & 4 & 4 & 5 \\
$v_{u}$ & 3 & 3 & 3 \\
$v_{f}$ & 3 & 5 & 3 \\
$v_{o+}$ & 12 & 12 & 12 \\
$v_{o-}$ & 10 & 4 & 5 \\
$v_{a}$ & 5 & 4 & 5 \\
$v_{F}$ & 3 & 6 & 3 \\
$v_{\Delta}$ & 12 & 15 & 12 \\
$E_{c}$ & 10 & 2 & 2 \\
\hline
\end{tabular}

* Best method for each parameter is italicized where significant difference is noted. See Appendix B for explanation of relative errors in $v_{0-}$ and in $v_{0+}$. at the slower components of calcium metabolism Harrison, McNeill, Finlay, Sheppard, and Mortimer (25) found pools with exponential rate constants reflected in plasma of $0.35,0.061$, and 0.0016 (day) $^{-1}$. The first two agree well with the last two constants of Neer et al. (24), i.e., 0.35 and 0.068 . Therefore, at present, at least five exponential components of calcium metabolism have been defined to describe the plasma calcium sp act curve and have rate constants of approximately $60,4,0.35,0.065$, and 0.0016 (day) $^{-1}$. Much work is needed to define which of these are important to skeletal metabolism and would best serve clinical and investigational needs.

In the present studies, sufficient data were not collected to define the very rapid component which is not necessary to determine the parameters of physiological interest. The commonly accepted two-component model for $\mathrm{Ca}_{e}$ was used to fit the first 10 days of data for each study phase, so as to be comparable to the majority of calcium kinetic work to date. The physiologic role of the fourth component is unclear and may be reflecting processes in slowly equilibrating bone more than in $\mathrm{Ca}_{e}$, as pointed out by Harris and Heaney (9), who also suggested that a two-component analysis currently appears most appropriate. Nevertheless, 
for purposes of comparison, 20 days of base line for each subject in the present work was also fit with a three-component exponential function [corresponding to components 2, 3, and 4 of Neer et al. (24)]. By doing this a much smoother fit was obtained for all of the data (see Fig. 3), even in the first portions of the curve, suggesting it may be necessary to consider at least 20 days of data and more than two components to represent $\mathrm{Ca}_{e}$ adequately.

Neer et al. (24) have pointed out that the physiologically important parameters of calcium metabolism, i.e., $v_{a}, v_{f}, v_{u}, v_{o_{-}}$, and $v_{o+}$ are independent of the configuration of pools used to describe $\mathrm{Ca}_{e}$. All of these parameters of calcium metabolism can be determined by combining any two of the three techniques discussed in this paper, balance, PTA, and/or CTA. Table $V$ compares estimated errors using different combinations of techniques. Errors of those parameters which appear to be significantly smaller by the use of one technique than by another are italicized. From these data, CTA and balance techniques apparently provide necessary and sufficient information to define the parameters of most physiologic interest with optimal precision. Furthermore, use of these two techniques can avoid the formulation of a model for $\mathrm{Ca}_{e}$ and the complex analysis required by PTA methods.

One of the advantages of determining $E_{c}$ from C'TA studies is its relative independence of model configuration for $\mathrm{Ca}_{e}$. That is not to say that the nature of $\mathrm{Ca}_{e}$ is not important in understanding skeletal kinetics. Nevertheless, using careful calcium balance and CTA techniques, $E_{c}$ and the other parameters of calcium metabolism including $v_{o_{-}}$can be obtained directly and precisely without resorting to sophisticated model construction and complex, nonlinear least-squares analysis of the data. The following relationship can be used to obtain $v_{t}$ for these calculations without P'TA data (see Appendix A for derivation):

$$
v_{t}=\frac{1}{\left(1-E_{c}\right)}\left[v_{I}-v_{F}+\frac{\left(X_{F}-X_{I}\right) v_{F}}{\left(1-X_{I}\right) E_{c}}\right] .
$$

Although in our CTA studies complex computer analysis was required to analyze the data, since tracer abundance was still about $3 \%$ from its asymptotic value even after 20 days, it is theoretically possible to arrive at an estimate of $E_{c}$ much more simply. Tracer may be added to the diet, at first in large quantities to get close to the asymptote and then at the constant level chosen with which to approach equilibrium. The average of the asymptotic abundance (AA) determinations in blood or urine (corrected for tracer from bone) made after a much shorter period of time than in our studies, could then be used to calculate $E_{c}: E_{c}=1-\mathrm{AA}$, where AA is expressed as a fraction of the dietary tracer abundance. Thus, com- bining this simplified technique with careful balance studies would yield optimal estimates of $v_{a}, v_{f}, v_{u}$, $v_{o-}, v_{o+}$, and $v_{t}$.

Physiological observations. The "model independent" parameters of calcium flow from phase I of this study are generally within the normal range reported by others (see Table II). The only exception is $v_{f}$ which is lower in the present studies. The large intersubject variation of calcium kinetic parameters observed by others $(23,24)$ is confirmed in this study, especially with regard to $v_{o_{+}}, v_{o_{-}}$, and $v_{u}$. Such wide variation makes normality of isolated calcium kinetic parameters difficult to establish. Serial studies in the same subject would undoubtedly be more sensitive and meaningful. Surprisingly, urinary calcium excretion in "normal" subjects apparently can be as much as $500 \mathrm{mg}$ day, as shown both in these studies and those of Neer et al. (24). Also significant negative calcium balances are often noted in "normal"subjects, in the present study as large as $-260 \mathrm{mg} /$ day and as large as $-175 \mathrm{mg}$ / day in the studies of Neer et al. (24). Since balances of this nature would almost certainly lead to skeletal disease, which in fact does not occur frequently in such normal subjects, one might consider the possibility of calcium balance cycling around a net balance of zero. Present evidence is not sufficient to rule out this possibility or to distinguish it from the effects of changes in the subjects' routines, although attempts were made to avoid altering these as much as possible.

A definite diurnal cycle in $v_{u}$ was noted in the subjects in this study. The cycle was suggestive with 12 -h urine collections from 6 a.m. to $6 \mathrm{p} . \mathrm{m}$. and $6 \mathrm{p} . \mathrm{m}$. to 6 a.m. However, 8 -h collections ( 6 a.m. -2 p.m., 2 p.m.10 p.m. and 10 p.m. -6 a.m.) showed a marked diminution in $\mathrm{v}_{u}$ by a factor of one-half to one-third during the nightly fasting period $(P<0.001)$, (see data in Appendix D.) This variation in $z^{\prime} u$ parallels creatinine clearance (26) and expected dietary absorption patterns allowing for gastrointestinal transit lags.

The acute effects (over a 15 day period) of intravenous calcium infusion on parameters of calcium kinetics are shown in Table I, B. D., phase I $a$. Definite decreases $(P \therefore 0.05)$ are noted in $v_{u}^{\prime}, v^{\prime} o_{-}$, and $E_{c}$, while other parameters remain unchanged, although there is a suggestion of a tendency for $i^{\prime}$ o+ to be elevated. The drop in $v_{u}$ with serum calcium levels of $9.5-11.0 \mathrm{mg}$ $100 \mathrm{ml}$ suggests the parathyroid glands were not totally suppressed by continuing, significant hypercalcemia; otherwise, low parathyroid hormone levels would decrease tubular reabsorption of calcium and or an elevated serum calcium would increase the filtered load of calcium, both of which would increase $v_{u}$. Calcium loading results in prolonged suppression of $\tau_{0-}$, possibly due to saturating sites of easily mobilizable calcium or "filling" sinks normally tending to lower serum 
calcium (e.g. uncalcified bone matrix). Thus, the "need" for parathyroid hormone to maintain normal blood calcium levels is decreased, leading to a decreased $v_{o-}$. Suppression of $v_{o_{-}}$by hypercalcemia stimulation of calcitonin activity has not been ruled out but is less likely in view of its short action. This evidence supports the results and clinically significant hypothesis of Pak et al. (27) and Jowsey, Hoye, Pak, and Bartter (28) that transient hypercalcemia may produce prolonged reduction of excessive bone breakdown.

In examining the effects of glucocorticoid therapy on parameters of calcium metabolism (see Table III), two facts become immediately apparent: $(a)$ urinary calcium is uniformly increased, and $(b)$ endogenous fecal excretion is uniformly decreased. However, changes in $v_{u}$ are much larger than in $v_{f}$, resulting in a large calcium loss from the body. Changes in other parameters of calcium metabolism are variable and apparently depend on the duration of corticoid administration. At least in the short term (the present study being conducted during the first 10 days of treatment) there is an elevation in $v_{a}$ noted in all four subjects; on the other hand, Wajchenberg, Pereira, Kieffer, and Ursic (23) noted a decrease in the fraction of dietary calcium absorbed after prolonged treatment (18 days) although their subjects varied their calcium intake between control and treatment periods and may have had an increased absolute value for $v_{a}$. Also, there appears to be an increase in $v_{t}$ with treatment without any significant change in pool size $\left(M_{1}+M_{2}\right)$ being noted. No significant change in $v_{o+}$ was apparent in the present studies, but Eisenberg (29) found it to be elevated after $6 \mathrm{wk}$ of glucocorticoid therapy; however, Eisenberg and Gordan (21) pointed out that accretion, $v_{o+}$, is decreased in Cushing's disease. Wajchenberg et al. (23) found an elevation in $v_{o-}$ and a decrease in balance $\left(v_{\Delta}\right)$ after 18 days of glucocorticoid administration. In the present studies, the one subject, (S. P.) showing an increased $v_{\sigma_{-}}$also had a decrease in $v_{\Delta}$ (balance); B. D. had a similar decrease in $v_{\Delta}$ but without increasing $v_{0-}$. However, no change in either $v_{o-}$ or $v_{\Delta}$ was noted in the other two subjects, possibly due to insufficient duration of treatment before study and/or the athletic nature of $\mathrm{P}$. $\mathrm{H}$. and the high maintenance estrogen dose of $\mathrm{E}$. W. It is interesting to note that $E_{c}$ decreased in all cases including S. P. in whom $v_{o-}$ increased, thus indicating that osteolysis decreased relative to the turnover rate. This observation suggests that the changes in $v_{0-}$ may be secondary to changes in $v_{t}$ brought on by increased urinary excretion of calcium and/or glucocorticoid tendency to induce hypocalcemia. The endocrine mechanism by which this might be accomplished could be a direct calciuretic and/or hypocalcemic effect of the glucocorticoid leading to slight parathyroid stimulation which in turn results in the observed changes in $v_{a}, v_{f}$, and $v_{0-}$. In any event, short-term glucocorticoid treatment did not increase the ratio of bone resorption to rapidly miscible pool turnover. Furthermore, except for the persistent calciuretic effect of glucocorticoids, the effects of these agents on other parameters of calcium metabolism are heavily dependent on the length of treatment.

It is fairly well established that androgens and estrogens decrease urinary calcium excretion and bone accretion $(29,30)$ while depressing $v_{0-}$ to an even greater extent and result in increased $v_{a}$ and $v_{\Delta}(9,31)$; furthermore, morphological techniques have also been used to demonstrate a reduction in bone resorption by sex hormones (32). However, the effects of such agents often take several weeks to manifest themselves as changes in skeletal calcium kinetics $(9,31)$. Thus, it is not surprising that few significant effects were found during the first 10 days of treatment with tetosterone or high dose Premarin in these studies. It is interesting to note that in two subjects, S. P. and P. H., $E_{c}$ did decrease although the decrease had not yet manifested itself as a decrease in $v_{o-}$, suggesting that $E_{c}$ may be a very sensitive indicator of effects on osteolysis relative to total turnover rate and that the primary effect of these agents on calcium metabolism is to decrease osteolysis. Coincidentally, it is in these same two subjects that a tendency to increase $v_{a}$ is also noted and in one of them, S. P., a significant decrease in $v_{f}$ is observed. Perhaps one reason no effect of increased estrogen administration was seen in E. W. was that her maintenance dose of $2.5 \mathrm{mg}$ Premarin per day was already maximally suppressing bone resorption as indicated by the general depression of all her parameters of calcium metabolism before the experimental period; effects of race and age cannot be ruled out as possible causes, however.

Summary. We have developed continuous tracer administration methods into a useful tool to more directly and precisely determine the rate of bone calcium resorption. A new parameter of calcium kinetics, $E_{c}$ or the fraction of rapidly exchangeable calcium pool turnover rate due to osteolysis, is defined and determinable with a standard error of less than $2 \%$ in each subject. The validity of these techniques is verified using classical PTA and calcium balance methods.

The results of 13 studies in four subjects lead to the following conclusions: First, there are large intersubject variations in parameters of in vivo calcium kinetics. Second, large urinary calcium excretion rates and significantly negative calcium balances are sometimes observed in normal, healthy adults, leading to the conclusion that serial studies in a given subject are much more valuable than isolated or grouped studies. Third, transient hyper- 
calcemia acutely and chronically results in a decreased rate of bone calcium resorption. Fourth, effects of androgens and estrogens on calcium kinetics require treatment for a prolonged period of time to observe suppression of osteolysis. And fifth, glucocorticoid therapy leads to increased calcium loss; in fact, acute glucocorticoid-induced hypercalciuria is the most striking and consistent effect of these agents, the other parameters of calcium metabolism being altered variably according to the duration of treatment.

\section{APPENDICES}

Appendix $A$. The equilibrium abundance of tracer, $E$, in $\mathrm{Ca}_{e}$ in CTA studies is the same as the abundance of tracer in the calcium flowing in to $\mathrm{Ca}_{e}$ :

$$
E=\frac{X_{I v_{a}}+X_{o-} v_{o-}}{v_{a}+v_{o}}
$$

where $X_{i}$ is the tracer abundance of source $i$ (I, dietary intake, $F$, fecal output, $o_{-}$, resorbing bone calcium) expressed as fraction of tracer abundance in nature. Therefore,

$$
v_{\sigma_{-}}=\frac{E-X_{I}}{X_{0-}-X_{I}} v_{t}
$$

since $v_{a}=v_{t}-v_{a}$.

Tracer abundance in serum and urine $\left(X=X_{s}=X_{u}\right)$ can be corrected for tracer coming from the diet. In the present experiments, since tracer is markedly reduced in the diet, a first approximation of the contribution of $X_{I}$ to $X$ at any time is $(1-X) X_{I}$, where $(1-X)$ represents an approximation of the fraction of calcium in pool I of dietary origin accumulated from the beginning of the study, and $X_{I}$ the abundance of tracer in the diet. Thus, $X$ may be corrected for tracer from diet:

$$
X_{c} \simeq X-(1-X) X_{I}
$$

This correction can be made precise by substituting $X_{c}$ for $X$ in the diet portion of the expression, i.e. $(1-X) X_{I}$ becomes $\left(1-X_{c}\right) X_{I}$, and solving for $X_{c}$ :

$$
X_{c}=\frac{X-X_{I}}{1-X_{I}}
$$

Using corrected data, $X_{c}$, the equilibrium abundance of tracer in $\mathrm{Ca}_{e}$ becomes $E_{c}$ reflecting the nondietary influx of tracer. Substituting into Eq. 9,

$$
v_{o-}=E_{c} v_{t}
$$

since $E=E_{c}, X_{o_{-}}=1.00$, and $X_{I}=0$ using dietcorrected data.

The turnover rate of $\mathrm{Ca}_{e}$ can also be calculated from $E_{c}$ and balance data. The following relationship comes from considerations of calcium balance with regard to the gastrointestinal system :

$$
v_{I}-v_{a}=v_{F}-v_{f}
$$

The terms $v_{I}$ and $v_{F}$ are known or measured directly and $v_{a}$ can be derived from Eq. 1 and 2:

$$
v_{a}=\left(1-E_{c}\right) v_{t}
$$

Considering tracer balance at equilibrium, $v_{f}$ can also be determined.

$$
X_{F} v_{F}=v_{f} E+\left(v_{I}-v_{a}\right) X_{I}
$$

Using Eq. 11 to calculate $E$ as a function of $E_{c}$, substituting $\left(v_{F}-v_{f}\right)$ for $\left(v_{I}-v_{a}\right)$ from Eq. 13 and then solving Eq. 15 for $v_{f}$ results in Eq. 16:

$$
v_{f}=\frac{\left(X_{F}-X_{I}\right) v_{F}}{\left(1-X_{I}\right) E_{c}} .
$$

Thus, by substituting from Eq. 14 and 16 into 13 an expression is obtained with $v_{t}$ as the only unknown which can be solved for according to the following expression :

$$
v_{t}=\frac{1}{\left(1-E_{c}\right)}\left[v_{I}-v_{F}+\frac{\left(X_{F}-X_{I}\right) v_{F}}{\left(1-X_{I}\right) E_{c}}\right] .
$$

Appendix B. As calculated from data in the present studies, the relative error in $v_{o+}$ is slightly larger than in $v_{o}$ using PTA and balance data. The disparity results because the magnitude of the errors for each of the three component determinations of $v_{0+}$ is about the same as for each of the four components of $v_{o_{-} \text {, }}$, while the larger value for $v_{o-}$ (due to the negative balances in the present studies) makes the percent error smaller. The component determinations may be derived as follows:

$$
\begin{aligned}
v_{o+} & =v_{t}-v_{f}-v_{u} ; \\
v_{o-} & =v_{o+}-v_{\Delta} \\
& =v_{t}-v_{f}-v_{u}-v_{I}+v_{F}+v_{u} \\
& =v_{t}-v_{f}+v_{F}+v_{I} .
\end{aligned}
$$

Thus, errors in the first two terms of each expression, $v_{t}$ and $v_{f}$, are identical; the errors in the third terms of each expression, $v_{u}$ and $V_{F}$, respectively, are similar using continuous fecal marking with chromium; and the error in $v_{I}$ is assumed to be negligible by experimental design.

Appendix $C$. The least-squares fits to the ${ }^{47} \mathrm{Ca}$ data for each subject during base-line phase $I$ are as follows with $X$ in units of fraction of administered dose per gram of calcium in $M_{1}$ at time $t$ in units of days.

Direct Measurement of Osteolysis in Man 
Two component analysis of the first 8-10 days :

$$
\begin{array}{ll}
\text { B. D. } & X=0.218 e^{-3.64 t}+0.175 e^{-0.135 t} \\
\text { S. P. } & X=0.348 e^{-3.38 t}+0.178 e^{-0.162 t} \\
\text { P. H. } & X=0.315 e^{-3.07 t}+0.144 e^{-0.165 t} \\
\text { E. W. } & X=0.311 e^{-2.42 t}+0.246 e^{-0.116 t}
\end{array}
$$

Three component analysis of the first 20 days:

B. D. $X=0.294 e^{-6.85 t}+0.104 e^{-0.427 t}+0.115 e^{-0.091 t}$

S. P. $X=0.405 e^{-7.96 t}+0.137 e^{-0.492 t}+0.107 e^{-0.108 t}$

P. H. $X=0.315 e^{-4.75 t}+0.112 e^{-0.404 t}+0.073 e^{-0.096 t}$

E. W. $X=0.308 e^{-9.40 t}+0.164 e^{-0.794 t}+0.207 e^{-0.094 t}$.

\begin{tabular}{|c|c|c|c|}
\hline Subject & 6 a.m.-2 p.m. & 2 p.m. -10 p.m. & 10 p.m.- 6 a.m. \\
\hline \multirow[t]{5}{*}{ B. D. } & 167 & 216 & 44 \\
\hline & 203 & 200 & 65 \\
\hline & 180 & 150 & 86 \\
\hline & 168 & 193 & 56 \\
\hline & 203 & 206 & 65 \\
\hline \multirow[t]{5}{*}{ S. P. } & 142 & 278 & 30 \\
\hline & 184 & 235 & 90 \\
\hline & 238 & 228 & 85 \\
\hline & 258 & 227 & 99 \\
\hline & 162 & 221 & 75 \\
\hline
\end{tabular}

Appendix $D$. Urinary calcium excretion (mg) during those portions of phase I where 8 -h collections were made. (No 8-h collections were made on P. H. or E. W.)

12-h urinary calcium excretion ( $\mathrm{mg}$ ) in these same two subjects during the 5 days before the above 8 -h collections.

\begin{tabular}{ccc}
\hline Subject & 6 a.m.-6 p.m. & 6 p.m.-6 a.m. \\
\hline B. D. & 209 & 205 \\
& 238 & 169 \\
& 203 & 149 \\
& 237 & 199 \\
& 191 & 149 \\
S. P. & 256 & 268 \\
& 273 & 201 \\
& 235 & 188 \\
& 293 & 247 \\
& 279 & 174 \\
\hline
\end{tabular}

\section{ACKNOWLEDGMENTS}

Aided by Grant 5 RO1 AM 13031 from the National Institutes of Health, and various intramural grants. Dr. Hansen was supported by a Bank of America-Giannini Foundation Fellowship.

\section{REFERENCES}

1. Frost, H. M. 1968. Tetracycline bone labeling in anatomy. Am. J. Phys. Anthropol. 29: 183.

2. Jowsey, J., P. J. Kelley, B. L. Riggs, A. J. Bianco, Jr., D. A. Scholz, and J. Gershon-Cohn. 1965. Quantitative microradiographic study of normal and osteoporotic bone. J. Bone Jt. Surg. A. Am. Vol. A47: 785.

3. Villanueva, A. R., Z. F. Jaworski, O. Hitt, P. Sarnsethsiri, and H. M. Frost. 1970. Cellular-level bone resorption in chronic renal failure and primary hyperparathyroidism. A tetracycline-based evaluation. Calcif. Tissue Res. 5: 288.

4. Cameron, J. R., and J. A. Sorenson. 1968. A reliable measurement of bone mineral content in vivo. Les Tissus Calcifiés, Ve Symposium Européen. G. Milhaud, M. Owen and H. J. J. Blackwood, editors. S. E. D. E. S. Soc. d'Édition d'Enseignement Supérieur, Paris. 343.

5. Aubert, J.-P. and G. Milhaud. 1960. Méthode de mesure des principales voies du métabolisme calcique chez l'homme. Biochim. Biophys. Acta. 39: 122.

6. Nordin, B. E. C., D. A. Smith, and J. Nisbet. 1964 Calculation of bone formation and resorption rates by continuous feeding of ${ }^{47} \mathrm{Ca}$. In Medical Uses of ${ }^{47} \mathrm{Ca}$ : Second Panel Report. International Atomic Energy Agency. STI/DOC/10/32, Vienna. 47.

7. Hansen, J. W., and G. S. Gordan. 1968. Direct measurement of osteolysis in the rat. Les Tissus Calcifiés, Ve Symposium Européen. G. Milhaud, M. Owen, and H. J. J. Blackwood, editors. S. E. D. E. S. Soc. d'Édition d'Enseignement Supérieur, Paris. 335.

8. Hansen, J. W. 1971. Direct Measurement of Osteolysis in Man. Doctoral Dissertation, University of California, San Francisco.

9. Harris, W. H., and R. P. Heaney. 1969. Skeletal renewal and metabolic bone disease. N. Engl. J. Med. 280: 193.

10. Corless, J. T. 1968. Isotopic abundance studies by neutron activation analysis. Summary Report NYO-3369-9, Jan. 15, 1968. Nucl. Sci. Abstr. no. 16415 22: 1708.

11. Bélanger, L. F., T. Semba, S. Tolnai, D. H. Copp, L. Krook, and C. Gries. 1966. The two faces of resorption. In Third European Symposium on Calcified Tissues. H. Fleisch, H. J. J. Blackwood, and M. Owens, editors. Springer-Verlag, K. G., Berlin. 1.

12. Riggs. B. L., Jr., J. B. Bassingthwaighte, J. Jowsey, and E. P. Pequegnat. 1970. Autoradiographic method for quantitation of deposition and distribution of radiocalcium in bone. J. Lab. Clin. Med. 75: 520.

13. Gordan, G. S., J. Hansen, and W. Lubich. 1966. Effects of hormonal steroids on osteolysis. Proc. 2nd. Int. Congr. Hor. Steroids, Excerpta Med. Found. Int. Congr. Seri. no. 132. P. 786.

14. Hargreaves, T., and G. A. Rose. 1965. The reproducibility of the balance method in man as applied to calcium and phosphorus. Clin. Sci. (Oxf.). 28: 537.

15. Heaney, R. P. 1963. Evaluation and interpretation of calcium-kinetic data in man. Clin. Orthop. Related. Res. 31 : 153.

16. Loken, H. F., J. S. Teal, and E. Eisenberg. 1963. Flame spectrophotometry of calcium with reversed oxyacetylene flame. Application to serum and urine. Anal. Chem. 35: 875.

17. Hansen, J. W., and S. G. Prussin. 1972. Precise measurement of microgram levels of ${ }^{48} \mathrm{Ca}$. Int. J. Appl. Radiat. Isot. 23 : 109.

18. Robertson, J. S., D. C. Tosteson, and J. L. Gamble, Jr. 1957. The determination of exchange rates in threc- 
compartment steady-state closed systems through the use of tracers. J. Lab. Clin. Med. 49: 497.

19. Heaney, R. P., and G. D. Whedon. 1958. Radiocalcium studies of bone formation rate in human metabolic bone disease. J. Clin. Endocrinol. Metab. 18: 1246.

20. Bauer, G. C. H., A. Carlsson, and B. Lindquist. 1957. Bone salt metabolism in humans studied by means of radiocalcium. Acta. Med. Scand. 158: 143.

21. Eisenberg, E., and G. S. Gordan. 1961. Skeletal dynamics in man measured by nonradioactive strontium. J. Clin. Invest. 40: 1809.

22. Massin, J. P. and G. Vallée. 1969. Étude isotopique du métabolisme calcique chez 10 sujets, selon le méthodes à 3 compartiments. II. Comparaison des analyses graphiques et par ordinateur. Ann. Endocrinol. 30: 417.

23. Wajchenberg, B. L., V. G. Pereira, J. Kieffer, and S. Ursic. 1969. Effect of dexamethasone on calcium metabolism and ${ }^{47} \mathrm{Ca}$ kinetics in normal subjects. Acta $61: 173$.

24. Neer, R., M. Berman, L. Fisher, and L. E. Rosenberg. 1967. Multicompartmental analysis of calcium kinetics in normal adult males. J. Clin. Invest. 46: 1364.

25. Harrison, J. E., K. G. McNeill, J. M. Finlay, R. H. Sheppard, and D. C. Mortimer. 1966. A preliminary study of human calcium metabolism using long-term whole body tracer measurements. Can. Med. Assec. J 94: 1092.

26. Pasternak, A., and B. Kuhlbäck. 1971. Diurnal variations of serum and urine creatine and creatinine. Scand. J. Clin. Lab. Invest. 27: 1.

27. Pak, C. Y. C., E. Zisman, R. Evens, J. Jowsey, C. S. Delea, and F. C. Bartter. 1969. The treatment of osteoporosis with calcium infusions. Clinical studies. Am. J Med. $47: 7$.

28. Jowsey, J., R. C. Hoye, C. Y. C. Pak, and F. C. Bartter. 1969. The treatment of osteoporosis with calcium infusions. Evaluation of bone biopsies. Am. J. Med. 47: 17.

29. Eisenberg, E. 1966. Effects of androgens, estrogens and corticoids on strontium kinetics in man. J. Clin. Endocrinol. Metab. 26: 566.

30. Gordan, G. S. 1971. Recent progress in calcium metabolism: clinical application. Calif. Med. 114: 28.

31. Heaney, R. P. 1968. Kinetic studies of calcium in metabolic bone disease. In Clinical Endocrinology. E. B. Astwood and C. E. Cassidy, editors. Grune and Stratton Inc. New York, 2nd edition. 309.

32. Riggs, B. L., J. Jowsey, P. J. Kelly, J. D. Jones, and F. T. Maher. 1969. Effect of sex hormones on bone in primary osteoporosis. J. Clin. Invest. 48: 1065. 